\title{
Synthesis, characterization and biodistribution of bisphosphonates Sm-153 complexes: correlation with molecular modeling interaction studies
}

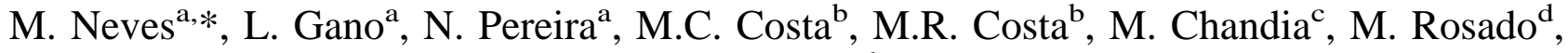 \\ R. Fausto ${ }^{\mathrm{d}}$ \\ anstituto Tecnológico e Nuclear, Sacavém, Portugal \\ ${ }^{\mathrm{b}}$ Instituto Nacional de Engenharia e Tecnologia Industrial, Lisbon, Portugal \\ ${ }^{\mathrm{c}}$ Chilean Nuclear Energy Comission, Santiago, Chile \\ ${ }^{\mathrm{d}}$ Department of Chemistry (CQC), University of Coimbra, Coimbra, Portugal
}

Received 26 May 2001; received in revised form 26 October 2001; accepted 11 November 2001

\begin{abstract}
Bisphosphonates (BPs) are characterized by a $\mathrm{P}-\mathrm{C}-\mathrm{P}$ backbone structure and two phosphonic acid groups bonded to the same carbon, and are established as osteoclast-mediated bone resorption inhibitors. The nature of the groups attached to the central carbon atom are responsible in determining the potency of bisphosphonates as anti-resorption drugs. However, it is not yet clear the exact relationship between their molecular structure and pharmacologic activities. In this study, molecular geometries of pamidronate, alendronate and neridronate, differing only in the length of the aliphatic chains, were predicted by molecular mechanics and their interactions with hydroxyapatite, the main bone mineral component, were examined. We report the synthesis and radiochemical characterization of ${ }^{153} \mathrm{Sm}$ complexes with pamidronate, alendronate and neridronate. Hydroxyapatite binding and biodistribution studies of these complexes have shown a good correlation with the theoretical molecular modeling interaction studies. So, it is possible to conclude that computational chemistry techniques are a good approach to evaluate specific interactions and may play a relevant role in determining the relative ability of BPs to mineral bone, and open new perspectives to the design of new BPs with increased pharmacological activity. These techniques could be extended to BPs as ligands to carrier radioactive metals, aiming for new bone therapeutic radiopharmaceuticals. (C) 2002 Elsevier Science Inc. All rights reserved.
\end{abstract}

Keywords: Bisphosphonates; Hydroxyapatite binding; Molecular modeling; Therapeutic radiopharmaceuticals

\section{Introduction}

Inorganic pyrophosphate, a naturally occurring polyphosphate and a known by-product of many biosynthetic reactions in the body, prevent calcification of soft tissues and regulates bone mineralization. Like pyrophosphate, bisphosphonates (BPs) have high affinity for bone mineral and were found to prevent calcification both in vitro and in vivo, and to inhibit the dissolution of hydroxyapatite crystals [9]. BPs are synthetic analogous of pyrophosphate in which the oxygen bridge has been replaced by a carbon atom with two attached side chains and are established as

* Corresponding author.

E-mail address: mneves@itn.pt (M. Neves). osteoclast-mediated bone resorption inhibitors. Some compounds exhibit short side chains, such as clodronate and etidronate; others have aliphatic chains of different lengths bearing terminal amino groups (pamidronate, alendronate, and neridronate) or substituted amino groups (olpadronate and ibandronate). Among the last generation of BPs with cyclic side chains, zoledronate with a nitrogen atom in an imidazole ring, is the most potent bisphosphonate described so far to inhibit bone resorption (Table 1). BPs, became the treatment of choice for a variety of bone diseases in which excessive osteoclast activity is an important pathological feature, including Paget's disease, metastatic and osteolytic bone disease, and hypercalcemia of malignancy $[8,28,29]$. BPs are also used as ligands to coordinate technetium-99m for bone scanning imaging [7] as methylene bisphosphonate (MDP), hydroxyethylene bisphosphonate (HEDP) and hy- 
Table 1

First and second bisphosphonates generation and their antiresorptive potency in acute in vivo models

\begin{tabular}{llll}
\hline Bisphosphonate & $\mathrm{R} 1$ & $\mathrm{R} 2$ & Potency* \\
\hline Clodronate & $\mathrm{Cl}$ & $\mathrm{Cl}$ & $\sim 10 \mathrm{x}$ \\
Etidronate & $\mathrm{OH}$ & $\mathrm{CH}_{3}$ & $\sim 1 \mathrm{x}$ \\
Pamidronate & $\mathrm{OH}$ & $\left(\mathrm{CH}_{2}\right)_{2} \mathrm{NH}_{2}$ & $\sim 100 \mathrm{x}$ \\
Alendronate & $\mathrm{OH}$ & $\left(\mathrm{CH}_{2}\right)_{3} \mathrm{NH}_{2}$ & $>100-<1000 \mathrm{x}$ \\
Neridronate & $\mathrm{OH}$ & $\left(\mathrm{CH}_{2}\right)_{5} \mathrm{NH}_{2}$ & $>100 \mathrm{x}$ \\
Olpadronate & $\mathrm{OH}$ & $\left(\mathrm{CH}_{2}\right)_{2} \mathrm{~N}_{\left(\mathrm{CH}_{3}\right)_{2}}$ & $>100-<1000 \mathrm{x}$ \\
Ibandronate & $\mathrm{OH}$ & $\left(\mathrm{CH}_{2}\right)_{2} \mathrm{~N}\left(\mathrm{CH}_{3}\right)\left(\mathrm{CH}_{2}\right)_{4} \mathrm{CH}_{3}$ & $>1000-<10,000 \mathrm{x}$ \\
Risedronate & $\mathrm{OH}$ & $\mathrm{CH}_{2}-3-$ pyridine & $>1000-<10,000 \mathrm{x}$ \\
Zoledronate & $\mathrm{OH}$ & $\mathrm{CH}_{2}$-imidazole & $>10,000 \mathrm{x}$
\end{tabular}

* From reference 8

droxymethylene bisphosphonate (HMDP) or to coordinate beta-emitter radionuclides (as samarium-153 and rhenium186) for bone pain palliation $[17,22]$.

In BPs, the groups attached to the central carbon atom determine their potency as anti-resorption drugs, thought it is not yet clear the precise relationship between their molecular structure and pharmacologic activity [8]. In order to shade light on the relationship between BPs molecular structure and hydroxyapatite- $-\mathrm{Ca}_{10}\left(\mathrm{PO}_{4}\right)_{6}(\mathrm{OH})_{2}-$ the main bone mineral component, we have carried out the following studies:

- a molecular modeling analysis, by molecular mechanics (MM), of the molecular structures of pamidronate, alendronate and neridronate-BPs differing only in the length of their aliphatic chains - and of their interaction with hydroxyapatite;

- the synthesis and chemical characterization of pamidronate, alendronate and neridronate;

- the synthesis and radiochemical characterization of ${ }^{153} \mathrm{Sm}$ complexes of the above BPs;

- in vitro hydroxyapatite binding and in vivo mice studies with the ${ }^{153} \mathrm{Sm}$-BPs complexes;

- establishment of a correlation between the computational studies and the in vitro and in vivo results.

\subsection{Mechanism of action of bisphosphonates}

In spite of many valuable contributions, the exact molecular mechanism by which BPs inhibit bone resorption, is not yet completely understood. Initially, BPs were supposed to prevent calcification by a strict physicochemical mechanism after adsorption to bone surfaces, but gradually become clear that cellular effects must be involved. Several authors suggest that the P-C-P moiety provides BPs with the ability to bind avidly to bone mineral in vivo and the anti-resorptive potency is critically dependent upon the structure of the side chains attached to the geminal carbon of the P-C-P moiety, and that the mechanism of action involves specific stereo interactions [28]. As osteoclasts are highly endocytic, BPs present in the resorption space may be internalized by endocytosis, and thereby affect their intracellular metabolism and induce apoptosis [29]. The uptake of BPs by osteoclasts in vivo has been confirmed using radiolabeled alendronate [21], which was internalized into intracellular vacuoles, and other subcellular compartments such as the cytoplasm, mitochondriae, and nuclei [28] and by the current used in nuclear medicine practice, MDP and HMDP labeled with Tc-99m for bone metastases imaging and Re-186/188 for bone palliation in patients with metastatic involvement [22].

For BPs intervenction at cellular level they should be presented at the osteoclast microenvironmental or resorption space, but it is not yet clear in which way their binding to the bone mineral matrix - hydroxyapatite-could affect the distinct potencies of the different BPs. They also shorten the life span of osteoclasts by inducing their programmed cell dead (apoptosis) and their inhibitory effects also may be mediated by other cells of the osteolineage or the macrophage family [11]. Recent in vitro studies indicate that BPs also inhibit proliferation, reduce viability and induce apoptosis in several human tumor cell lines. In addition, BPs reduce the invasion of tumors cells through extracellular matrix and impar the binding of tumor cells to bone in vitro. BPs may have the potential to exert direct antitumor effects in vivo, particularly in bone metastases where the local concentration is elevated by the enhanced osteoclastic resorption of BP-loaded bone [12]. The molecular mechanisms by which tumor cells metastasize to bone are likely to involve invasion, cell adhesion to bone, and release of soluble mediators from tumor cells that stimulate osteoclastmediated bone resorption. According to Boissier et al., 2000, [3], BPs pretreatment of breast and prostate carcinoma cells inhibited tumor cell invasion in a dose-dependent manner, and the order of potency of the BPs under study follows the same order of antiresorptive potency (see Table 1). Also, Fromigue et al., 2000, [11] reported that clodronate, pamidronate, ibandronate and zoledronate can inhibit breast cancer cell growth, namely MCF-7, T47D, and MDA.MB.231 cell lines by inducing apoptosis or primary necrosis depending on the cell type and the BPs concentration. The relative percentages of viable, apoptotic, and necrotic cells to the total number of cells scored, follows also the relative order of antiresorptive potency, i.e. zoledronate $>$ ibandronate $>$ pamidronate $>$ clodronate. So, BPs as antiresorptive agents or as cells growth inhibitors follow identical relative order of potencies. From preclinical studies with zoledronic acid and other BPs Mundy et al., 2001, [24], conclude that bisphosphonate-associated tumor reduction in bone is most likely mediated by osteoclast inhibition or is related to high local concentration of BPs in the bone compartment. It is interesting to note that BPs inhibit tumor cells invasion in a dose-dependent manner, and that apoptosis and necrosis of breast cancer cells are depending of BPs concentration. If biologic effects are depending of BPs concentration, it could mean that their affinity to the bone matrix plays a fundamental role. So, it is possible to admit that two conjugate effects- hydroxyapa- 
tite BPs binding and cellular interactions-would occur. These two possible conjugate effects are supported by the work of Shakespeare W. et al., 2000, [31], in which a nonpeptide inhibitor (AP22408) with a bisphosphonate moiety demonstrates a remarkably hydroxyapatite affinity, and is the tighest binding inhibitor for Src SH2 (targeted disruption of the Src gene in mice has revealed a critical role of Src in the osteoclats function) and shows in vitro antiresorptive properties in an osteoclast-mediated cellular assay and in vivo TPTX model of parathyroid hormone-induced bone resorption. Two negative controls, (a bone-targeted analog that does not bind to $\mathrm{Src} \mathrm{SH} 2$ and a nonbonetargeted analog that has high affinity to bind Src SH2) in an rabbit osteoclast resorption assay validate the AP22408 biological properties. It seems that both, bone-targeted via hydroxyapatite binding and $\mathrm{Src} \mathrm{SH} 2$ binding are essential. Then, two mechanims could be outlined:

(i) BPs will bind to the bone mineral matrix and the inhibition resorption will be the result of their interaction with cell(s) from the intracellular environment,

(ii) BPs will bind to the mineral matrix and it will induces morphologic changes that lead to diminished crystal growth and may prevent the adhesion of cells from extracelular environment.

These fact justifies the interest to structurally characterize the different BPs, since the peculiarities of their substituted groups shall correlate with their relative potencies as powerful inhibitors of the osteoclast activity and as potential inhibitors of specific cells growth.

\subsection{Technetium, rhenium and lanthanide bisphosphonates complexes}

The exact molecular structure of technetium, rhenium and lanthanide bisphosphonates complexes are not known. The tendency of bisphosphonates as ligands, to form polymeric metal complexes complicates their isolation and characterization. In general, the experimental characterization of the molecular structure of technetium compounds is only possible using the carrier radionuclide Tc-99 and, depending on the reaction conditions (e.g. pH, metal concentration, presence or absence of oxygen, type of reducing agent), the reduced technetium center exist in a variety of oxidation states, mixed metal complexes, uncommon coordination numbers, and hydrolyzed, colloidal, dimeric and polymeric forms. Indeed, taken in consideration the complexity of the technetium chemistry, it is easy to understand that the translation of the results obtained at carrier level (Tc-99) to the chemistry at no-carrier level (Tc-99m), is not a straightforward procedure and represents a challenge to investigation. Attempts to characterize Tc-99 bisphosphonates complexes by gel chromatography, HPLC, amperometric titrations, polarography, etc., reveal a mixture of several complexes, which are produced in relative amounts and yields depend- ing mainly on the $\mathrm{pH}$ and oxygen content ( 7 and references wherein). Reversed-phase HPLC of Tc-99m bisphosphonates have shown also complicated mixtures of several radiochemical species [23]. Libson et. al. [20] have isolated a Tc-99-MDP polymeric complex with an approximate octahedral coordination. Each MDP ligand bridges two symmetry Tc atoms, and each Tc atom is bound to two symmetry MDP ligands. Similar problems would occur with rhenium, whose chemistry is closed related to technetium, and there is not any available information on rhenium coordination complexes with bisphosphonates. On the other hand, a series of lanthanides complexes with HEDP (1hydroxyethane-1,1-diphosphonic acid) were studied by Nash K. L. et al. [26]. Three different structural variations of the 1:2 complexes have been observed:

- Nd crystallizes as a heptahydrate with an eight-coordinate square antiprismatic structure, $\left[\mathrm{Nd}\left(\mathrm{H}_{3} \mathrm{HEDP}\right)\right.$ $\left.\left(\mathrm{H}_{2} \mathrm{HEDP}\right)\right] .7 \mathrm{H}_{2} \mathrm{O}$, the metal ion being coordinated in a linear polymeric fashion to four HEDP ligands via $\mathrm{P}=\mathrm{O}, \mathrm{P}_{-} \mathrm{O}^{-}$and one $\mathrm{P}-\mathrm{OH}$ moiety,

- Eu complex $\left[\mathrm{H}_{3} \mathrm{O}\right]\left[\mathrm{Eu}\left(\mathrm{H}_{2} \mathrm{HEDP}\right)_{2}\right] \cdot 12 \mathrm{H}_{2} \mathrm{O}$, is also an eight-coordinate polymeric structure and has the highest symmetry of any of the complexes characterized,

- the heavy lanthanides $(\mathrm{M}=\mathrm{Tb}, \mathrm{Dy}, \mathrm{Ho}, \mathrm{Er}, \mathrm{Tm}, \mathrm{Yb}$, $\mathrm{Lu}, \mathrm{Y}$ ) form an isostructural series of compounds in a seven-coordinate capped trigonal prismatic geometry $\left[\mathrm{M}\left(\left(\mathrm{H}_{3} \mathrm{HEDP}\right)\left(\mathrm{H}_{2} \mathrm{HEDP}\right)\right] .5 \mathrm{H}_{2} \mathrm{O}\right.$. The overall coordination to four HEDP ligands in a linear polymer, is comparable to that for the $\mathrm{Nd}$ and $\mathrm{Eu}$ complexes, except that the seven-coordinate metal ions release one $\mathrm{P}-\mathrm{O}$ site which coordinates the fifth hydrogen ion.

The extensive intramolecular hydrogen bonded network and water molecules sequestration is consistent with the previous observation of low complexation entropy and exothermic heats, for europium BPs complexes [25].

\subsection{Calcium affinity by metal-bisphosphonates complexes}

The chemistry of bone imaging agents ultimately resides in the high calcium affinity of the bisphosphonates even after they are coordinated to a metal. To a first approximation, it is the bisphosphonate ligand, and not the nature of the bisphosphonate-metal complex, that determines the biodistribution. This is evidenced by the fact that HEDP labeled with P-32, HEDP complexed to Sn-113, HEDP complexed to Re-186 and HEDP-Tc-99m complexes prepared by reduction of pertechnetate with $\mathrm{Sn}(\mathrm{II}), \mathrm{NaBH}_{4}$ and $\mathrm{Cu}(\mathrm{I})$, all accumulate in bone. Thus, the HEDP-metal complexes bone uptake is not dependent on the nature of metal center, but is primarily driven by the nature of the ligand ( 7 and references herein). In fact, the HEDP-metal complexes $(\mathrm{M}=\mathrm{Sn}, \mathrm{Re}, \mathrm{Tc}, \mathrm{Cu})$ have not exactly all the same bone uptake, but deviations could be explained by the different complexes involved, which have distinct chemical and 
physical natures. According to Jurisson et al. [15] the apparent equilibrium constants, governing the association of calcium (II) with several coordinated bisphosphonate ligands are large, ranging from $4 \times 10^{3}$ to $2 \times 10^{6} \mathrm{M}^{-1}$, showing that even after coordination the bisphosphonate ligand has considerable affinity for calcium. The calcium affinity of ligands as $\left(\mathrm{O}_{3} \mathrm{P}-\mathrm{C}(\mathrm{R})\left(\mathrm{R}^{\prime}\right)-\mathrm{PO}_{3}{ }^{4-}\right)$ depends strongly on the nature of the $\mathrm{R}$ and $\mathrm{R}^{\prime}$ groups, the observed variations result from steric, electronic and chelate effects. Binding to calcium of a coordinated bisphosphonate can occur not only through the common $\mathrm{PO}_{3}$ groups but also through to the specific $\mathrm{R}$ groups. This additional binding mode converts the bisphosphonate ligand from a bidentate to a tridentate bridge, which it is expected to enhance the calcium affinity of the coordinated bisphosphonate. Some authors [13], claim that in vitro studies of ${ }^{99 \mathrm{~m}} \mathrm{Tc}$-BPs complexes adsorption on calcium phosphate are important as a pre-evaluation of bone uptake. It was also possible to establish a rationale correlation between adsorption properties of radioactive metal BPs complexes and the structures of the free ligands, as MDP, HMDP and HEDP labeled with C-14 [10].

\section{Molecular modeling}

Computer-assisted molecular design has become a key component on the elucidation of the relationship between molecular structure and function of biological systems. Computer modeling allows the generation and evaluation of physicochemical properties of molecular systems and, by simulation of intermolecular interactions, can be used to recognize potential sites and lead to the understanding of biological phenomena. Molecular recognition at the interface between inorganic materials and organic templates is known to be a powerful force in the control of crystalization and mineralization in both synthetic and biologic systems. For example, the interaction between alkyldiphosphonates and the surfaces of barium sulfate was studied at molecular level using molecular modeling techniques, in order to establish a reliable mechanism for crystal growth inhibition by molecules [2]. The relative effectiveness of phosphonate retarders on hydrating cements was also evaluated by computational methods through the simulation of specific interactions between several phosphonates and a surface layer of ettringite [6]. In the present study, the interactions between three BPs differing only in the length of their aliphatic chain: (pamidronate $-\left(\mathrm{CH}_{2}\right)_{2}-\mathrm{NH}_{2}$; alendronate $\left(\mathrm{CH}_{2}\right)_{3}-\mathrm{NH}_{2}$; neridronate $\left.-\left(\mathrm{CH}_{2}\right)_{5}-\mathrm{NH}_{2}\right)$ and an infinite periodic surface of hydroxyapatite, were evaluated by molecular modeling using the second generation CVFF 950 (version 1.01) force field.

Prior to simulate the interactions of the BPs with hydroxyapatite, a detailed study of the preferred conformations (a three-dimensional arrangement of atoms) assumed by the isolated molecules of the studied BPs was under- taken. This study provided the necessary basic knowledge on the molecular structure of the BPs, thus enabling the further later identification of the main structural changes suffered by the BPs due to the interaction with hydroxyapatite. Among the various available methods to compute molecular structures, molecular mechanics (MM) is able to predict structures with high accuracy and little computational expense, even for large molecular systems [4]. Basically, MM assumes that the energy (E) of a given molecule can be described as a sum of energies associated with distortions of its different internal mechanical degrees of freedom (bond stretchings, $\mathrm{E}_{\nu}$, angle bendings, $\mathrm{E}_{\delta}$, torsions, $\mathrm{E}_{\tau}$, changes in non-bonded distances $\mathrm{E}_{\mathrm{nb}}$ ) from their "natural geometry" and specific electrostatic (Coulomb type, $\mathrm{E}_{\mathrm{C}}$ ) interactions (additional terms can be added to consider specific interactions, like, for instance, hydrogen-bonding):

$$
\mathrm{E}=\mathrm{E}_{\nu}+\mathrm{E}_{\delta}+\mathrm{E}_{\tau}+\mathrm{E}_{\mathrm{nb}}+\mathrm{E}_{\mathrm{C}}+\ldots
$$

E is sometimes named "steric energy" [27], and it represents the difference between the energies of the real molecule and the hypothetical molecule where all the structural parameters assume their "natural values". The whole set of functions used to describe the different energy contributions is named "force field" and this is strongly dependent on both the analytical form of the potential energy function used to represent a given type of contribution and the particular set of parameters defining each particular interaction of a given type (e.g., C-C or C-O stretching; C-O-C or C-C-C bending, $\mathrm{C}-\mathrm{C}$ or $\mathrm{C}-\mathrm{N}$ torsion, etc.). Experience has shown that the choice of functions in the force field is critical. In addition, while a variety of efficient and fast computational algorithms are widely available, the final force field should be both reliable and simple. In the present study, the secondgeneration CVFF 950 (version 1.01) force field of Hwang and co-workers [14] was selected for calculations. These were made using the Cerius-2 system of programs [5] running in a Silicon Graphics $\mathrm{O}_{2}$ workstation.

\section{Material and methods}

\subsection{General}

The ligands pamidronate, alendronate and neridronate are prepared according to the general procedure to linear aliphatic BPs [18] and characterized by elemental analysis, ${ }^{1} \mathrm{H}-\mathrm{NMR}$ and ${ }^{13} \mathrm{C}$-NMR. ${ }^{153} \mathrm{Sm}$ was produced in the Portuguese Nuclear Reactor at a neutron flux of $2 \times 10^{13}$ n. $\mathrm{cm}^{-2} \cdot \mathrm{s}^{-1}$ from samarium oxide enriched targets $98 \%$ (Campro Scientific). Hydroxyapatite Bio-Gel ${ }^{\circledR}$ HTP Gel is a Bio-Rad Laboratories product. Unless otherwise indicated all materials used were reagent grade. 


\subsection{Ligand synthesis}

The ligands pamidronate, alendronate and neridronate $(\mathrm{n}=2,3,5)$, were prepared from the corresponding carboxylic acid percursors according to the following reaction:

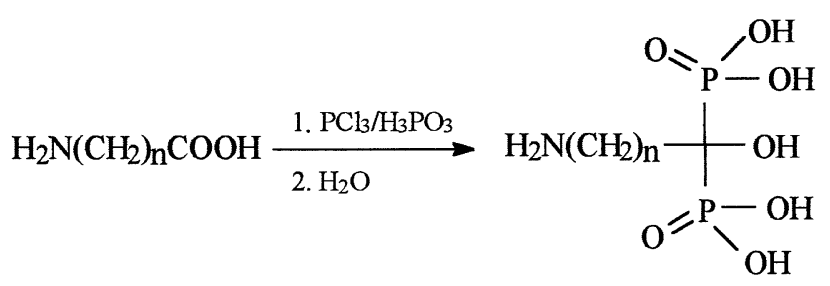

Alendronate monosodium trihydrate $-\left(\mathrm{C}_{4} \mathrm{H}_{12} \mathrm{~N} \mathrm{NaO}_{7} \mathrm{P}_{2} \cdot 3 \mathrm{H}_{2} \mathrm{O}\right)$ :

To aminobutanoic acid $\left(2 \mathrm{~g}, 1.9 \times 10^{-2} \mathrm{~mol}\right)$ in a three-necked round-bottom flask $\mathrm{H}_{3} \mathrm{PO}_{3}(1.6 \mathrm{~g}, 1.9 \times$ $\left.10^{-2} \mathrm{~mol}\right)$ was added under inert atmosphere followed by $8 \mathrm{~mL}$ of methanesulfonic acid. After heating at $65^{\circ} \mathrm{C}$, $\mathrm{PCl}_{3}\left(3.5 \mathrm{~mL}, 4 \times 10^{-2} \mathrm{~mol}\right)$ was added and the reaction allowed to proceed for 20-22 hours. The resulting yellow viscous reaction mixture was cooled to room temperature and quenched with $30 \mathrm{~mL}$ of ice-cold water. The $\mathrm{pH}$ was adjusted to 4.3 with $\mathrm{NaOH}$ solution and the white precipitate formed was collected by filtration, and then air and vacuum dried at $40^{\circ} \mathrm{C}$, to give alendronate monosodium trihydrate $(4.39 \mathrm{~g}, 70 \%)$, as white crystals, m.p. $270-274^{\circ} \mathrm{C} ;{ }^{1} \mathrm{H}$ NMR $\left(\mathrm{D}_{2} \mathrm{O}\right): \delta 2.97(2 \mathrm{H}, t), 1.93(4 \mathrm{H}, t)$, ${ }^{13} \mathrm{C}$ NMR $\left(\mathrm{D}_{2} \mathrm{O}\right): \delta 39.97,30.56,22.10(\delta)$; Elemental Analysis for $\mathrm{C}_{4} \mathrm{H}_{12} \mathrm{NNaO}_{7} \mathrm{P}_{2} .3 \mathrm{H}_{2} \mathrm{O}, \%$ Calculated: $\mathrm{C}$ 14.78, H 5.58, N 4.31; Found: C 14.70, H 5.63, N 4.11. Pamidronate and neridronate were prepared in a similar manner following the procedure above described for alendronate by using $\beta$-alanine and 6 -aminocaproic acid respectively as starting materials. Physical and spectral data obtained were as follows:

Pamidronate monosodium monohydrate- $-\left(\mathrm{C}_{3} \mathrm{H}_{10} \mathrm{~N}\right.$ $\left.\mathrm{NaO}_{7} \mathrm{P}_{2} .0 .5 \mathrm{H}_{2} \mathrm{O}\right)$ : as a white solid $(5.48 \mathrm{~g}, 35 \%):{ }^{1} \mathrm{H}$ NMR $\left(\mathrm{D}_{2} \mathrm{O}\right): \delta 3.32(2 \mathrm{H}, t, J=6.9 \mathrm{~Hz}), 2.27(2 \mathrm{H}, m)$; ${ }^{13} \mathrm{C}$ NMR $\left(\mathrm{D}_{2} \mathrm{O}\right): \delta 73.66\left(t, J_{C-P n}=132 \mathrm{~Hz}\right), 37.06$, $31.50(t)$; Elemental Analysis for $\mathrm{C}_{3} \mathrm{H}_{10} \mathrm{NNaO}_{7} \mathrm{P}_{2} \cdot \mathrm{H}_{2} \mathrm{O}$, $\%$ Calculated: C 13.10, H 4.40, N 5.09; Found: C 12.85 , H 4.34, N 4.83.

Neridronate monosodium monohydrate- $-\left(\mathrm{C}_{6} \mathrm{H}_{16} \mathrm{~N}\right.$ $\mathrm{NaO}_{7} \mathrm{P}_{2} \cdot 0.5 \mathrm{H}_{2} \mathrm{O}$ ): as a white solid (3.99 g, 42\%): m.p. $263-266^{\circ} \mathrm{C},{ }^{1} \mathrm{H}$ NMR $\left(\mathrm{D}_{2} \mathrm{O}\right): \delta 3.05(2 \mathrm{H}, t, J=7.6 \mathrm{~Hz})$, 1.90-2.05 (2H, m), 1.61-1.79 (4H, m), $1.44(2 \mathrm{H}, m) ;{ }^{13} \mathrm{C}$ $\operatorname{NMR}\left(\mathrm{D}_{2} \mathrm{O}\right): \delta 74.92\left(t, J_{C-P n}=132 \mathrm{~Hz}\right), 40.06,36.00$, 26.81, 26.66, $26.36\left(t, J_{C-P n}=6.8 \mathrm{~Hz}\right)$; Elemental Analysis for $\mathrm{C}_{6} \mathrm{H}_{16} \mathrm{NNaO}_{7} \mathrm{P}_{2} .0 .5 \mathrm{H}_{2} \mathrm{O}, \%$ Calculated: $\mathrm{C} 23.39, \mathrm{H}$ 5.56, N 4.55; Found: C 22.90 H 5.65 N 4.39.

\subsection{Synthesis and characterization of ${ }^{153} \mathrm{Sm}$-BPs complexes}

The reactions were conducted according to the procedure described by Zeevaart et al. [33] for ${ }^{166} \mathrm{Ho}$-pamidronate. To each ligand in alkaline solution, was added the required amount of the radioactive solution in chloride form $\left({ }^{153} \mathrm{SmCl}_{3}\right)$, according to a ligand/metal molar ratio ranging from 110 to 170 , and the $\mathrm{pH}$ ajusted to 8-9. Complexation of the radioactive metal, was assessed by ascending chromatography, using ITLC-SG (Gelman Sciences Inc., Ann Arbor, MI) strips developed with $\mathrm{H}_{2} \mathrm{O} / \mathrm{NH}_{4} \mathrm{OH}$ (25:1) (A system) and acetone $/ \mathrm{H}_{2} \mathrm{O} / \mathrm{HCl}$ (7:2:1) (B system). The profiles of the peaks were drawn using a Bioscan AR-2000 scanning equipped with a scintillation probe.

\subsection{Hydroxyapatite binding}

To vials containing 5.0, 10.0, 15.0, 20.0, 25.0 and 50.0 $\mathrm{mg}$ of hydroxyapatite, were added $2 \mathrm{~mL}$ of physiological saline solution at $\mathrm{pH}=7.4$ and the mixtures shaken for 24 hours at $37^{\circ} \mathrm{C}$ into a water-bath. Then, $50 \mu \mathrm{L}$ of each radioactive preparation was added and the mixtures shaken for 24 hours at $37^{\circ} \mathrm{C}$. All suspensions were filtered through a $0.2 \mu \mathrm{m}$ Millipore filter, centrifugated and two aliquots of $100 \mu \mathrm{L}$ were taken from each vial and the radioactivity measured into a DPC Gamma-C12 counter, calibrated for ${ }^{153} \mathrm{Sm}$. Blanks were also prepared in the same experimental conditions, except the presence of hydroxyapatite. Six vials were assayed for each preparation. The percentage binding to hydroxyapatite (HB) was calculated by $\mathrm{HB}=(1-$ $\mathrm{A} / \mathrm{B}) \times 100$, being $\mathrm{A}$ the mean value of the measured radioactivity of samples under study and $B$ the mean value of blanks.

\subsection{Animal studies}

The animal experimentation was performed in accordance with the National Code for the Handling and Use of Animals in Research, Education, Diagnosis and Testing of Drugs and Related Substances (which conforms to the internationally accepted standards). Quantitative tissue distributions of the ${ }^{153} \mathrm{Sm}$-BPs complexes were evaluated in groups of six female mice (randomly-bred Charles-River, Wilmington, MA), weighting approximately 25 grams each. Animals were intravenously injected with $100 \mu \mathrm{L}$ (5-10 $\mathrm{MBq}$ ) of each radioactive preparation via the tail vein and were maintained on a normal diet ad libitum. The injected dose was measured using a calibrator. At 1, 3 and 24 hours after injection the animals were sacrificed by cervical dislocation, and weighted. Femurs were removed, weighted and assayed by gamma counter (DPC Gamma-C12). Skeletal uptake was determined assuming that bone represents $10 \%$ of all body weight. Thus, radioactivity in all bone was calculated from the femur radioactivity uptake. The results are expressed as percent of injected dose (\% ID). 

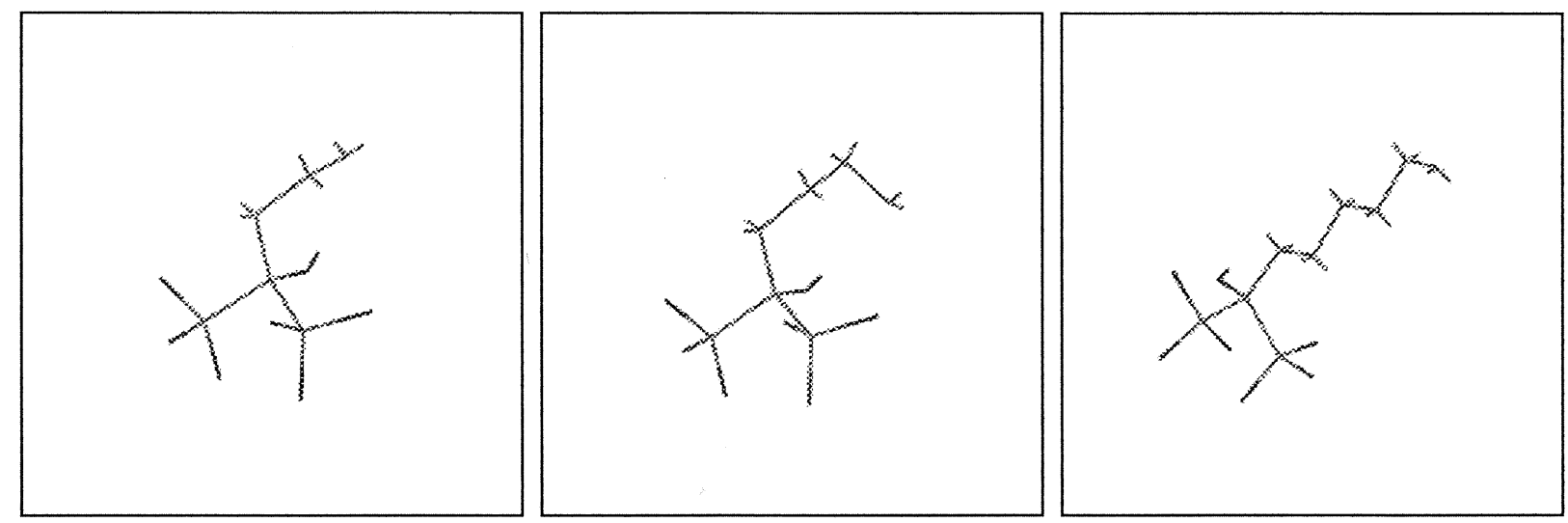

A

B

C

Fig. 1. Molecular geometries of the most stable conformers of pamidronate (A), alendronate (B) and neridronate (C) (isolated molecule in vacuum).

\section{Results and discussion}

\subsection{Molecular modeling}

In order to test the performance of the selected force field, we started the calculations by modeling the structure of alendronate and comparing the resulting geometry with that obtained experimentally [32]. Even though the properties being compared are not exactly equivalent (experimental structure refers to the crystalline state, whereas the calculated structure corresponds to the isolated molecule equilibrium geometry), the theoretical and experimental data show a fairly good agreement. Since the calculations were able to reproduce satisfactorily the experimental geometry for alendronate, it was assumed that the structures of pamidronate and neridronate could also be estimated reasonably (Fig. 1). The different conformational states of the three BPs studied were obtained by multiple minimization of randomly generated initial structures. The number of initial structures generated was 1000 with all torsional angles let free to vary in the optimization procedure. For pamidronate, 10 different conformers with energies within 5 $\mathrm{kcal} \mathrm{mol}^{-1}$ relatively to the global minimum were obtained as a result of this process, while for alendronate and neridronate the calculations predict, respectively, 34 and 171 different conformers within this range of energies (Fig. 2). The large increase in the number of conformers with the length of the carbon chain could be anticipated, since the conformational flexibility must increase with the number of torsional degrees of freedom in the molecule. For the three molecules, the density of states was found to increase significantly with the energy. Indeed, the energy difference between the first excited and the ground conformational states in pamidronate is $\Delta \mathrm{E}^{*(1-10)} \approx 1.5 \mathrm{kcal} \mathrm{mol}^{-1}$, while the next 8 excited conformational states have energies differing only by ca. $1.8 \mathrm{kcal} \mathrm{mol}^{-1}\left(\Delta \mathrm{E}^{*(10-2)}\right)$. For alendro- nate, $\Delta \mathrm{E}^{*(1-0)} \approx 0.9 \mathrm{kcal} \mathrm{mol}^{-1}, \Delta \mathrm{E}^{*(10-2)} \approx 1.2 \mathrm{kcal} \mathrm{mol}^{-1}$ and $\Delta \mathrm{E}^{*(34-11)} \approx 1.8 \mathrm{kcal} \mathrm{mol}^{-1}$, while for neridronate $\Delta \mathrm{E}^{*(1-0)} \approx 0.3 \mathrm{kcal} \mathrm{mol}^{-1}, \Delta \mathrm{E}^{*(10-2)} \approx 0.7 \mathrm{kcal} \mathrm{mol}^{-1}$, $\Delta \mathrm{E}^{*}(34-11) \approx 0.7 \mathrm{kcal} \mathrm{mol}^{-1}$ and $\Delta \mathrm{E}^{*(171-35)} \approx 2.8 \mathrm{kcal}$ $\mathrm{mol}^{-1}$ (see also Fig. 2). It can then be concluded that for the isolated BPs, the most stable conformer assumes a particular importance from a practical point of view, thus justifying a more detailed analysis of its structure.

For both pamidronate and alendronate, the most stable conformer is characterized by the presence of an intramolecular $\mathrm{OH} \ldots \mathrm{N}$ hydrogen bond. The establishment of this bond leads to stabilization of gauche conformations of the carbon chain, in order to ensure the most favorable hydrogen bond interaction geometry. In the case of neridronate, the global minimum was found to correspond to a conformer that shows an all-trans (zig-zag) carbon chain. This different conformational behavior can be easily understood taking into consideration that hydrocarbon chains assume preferentially this type of conformation unless other intramolecular interactions can compensate the increase of energy due to the presence of gauche conformations around the $\mathrm{C}-\mathrm{C}$ bonds. So, while in the case of pamidronate and alendronate, the presence of the intramolecular hydrogen bond can compensate energetically the destabilization due to the gauche arrangements necessary to obtain the most favorable hydrogen bond interaction geometry, in the case of neridronate, the stabilization due to the intramolecular hydrogen bonding is not enough to compensate the increase of energy associated with the gauche arrangements.

Once the main structural trends in the BPs were established, the intermolecular interactions between these molecules and hydroxyapatite could be examined. To do this, the molecule of each BP was placed in the vicinity of the (001) Miller plane of the crystal of hydroxyapatite, which has been described previously as the most favorable growing 


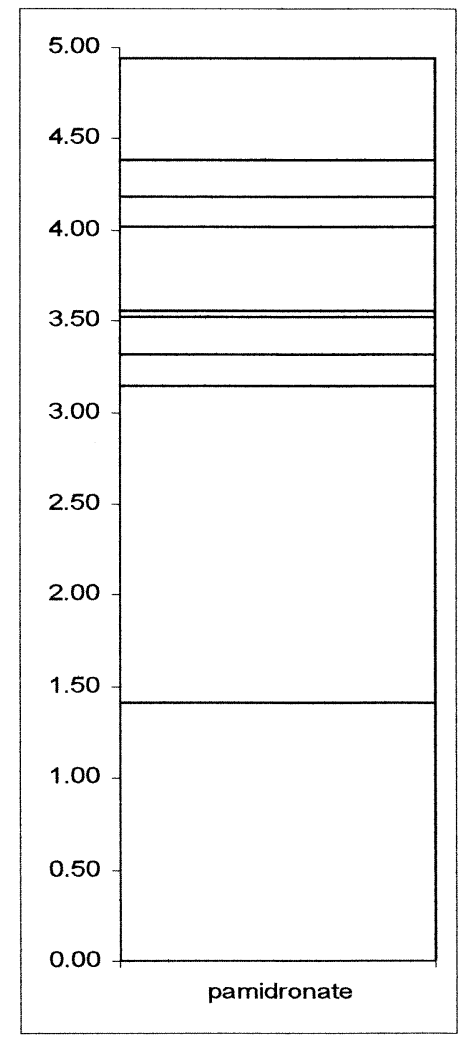

A

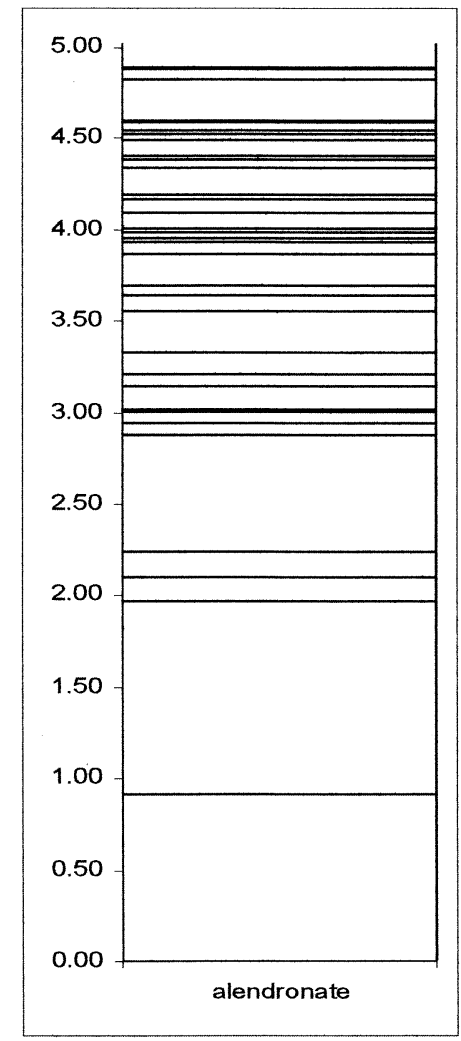

B

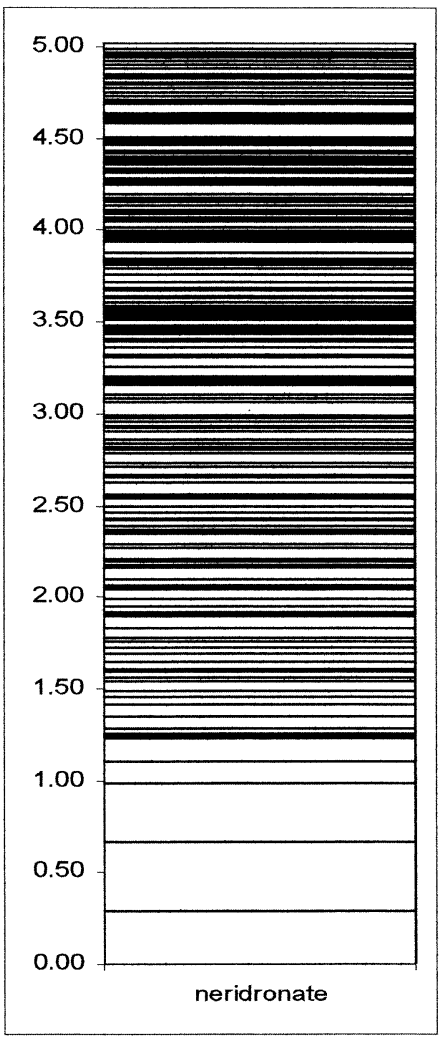

C

Fig. 2. Conformational energy levels $\left(\mathrm{kcal} \mathrm{mol}^{-1}\right)$, relative to the conformational ground state for pamidronate (A), alendronate (B) and neridronate (B).

surface of hydroxyapatite [1]. The crystalline structure of hydroxyapatite reported by Kay and Young [16] was used in the simulations. Hydroxyapatite crystal is constituted by layers of phosphate and hydroxide groups intercalated with layers of calcium ions. The (001) surface of the hydroxyapatite crystal, shows that each hydroxyl group is surrounded by six calcium atoms, three in one plane and the remaining three in an adjacent plane (Fig. 3).

In order to obtain the minimum energy BP-hydroxyapatite complex, a systematic search on the potential energy surface of the BP-hydroxyapatite (001) surface complex was performed. The structure of hydroxyapatite was considered fixed during the energy minimization process while geometries of the BPs were fully optimized. For each BP, from the whole set of complexes obtained by this way, the lowest energy species was selected for further analysis. The minimum energy hydroxyapatite complexes of the three BPs studied are shown in Fig. 4.

From the results presented in Fig. 4, the following main conclusions could be drawn:
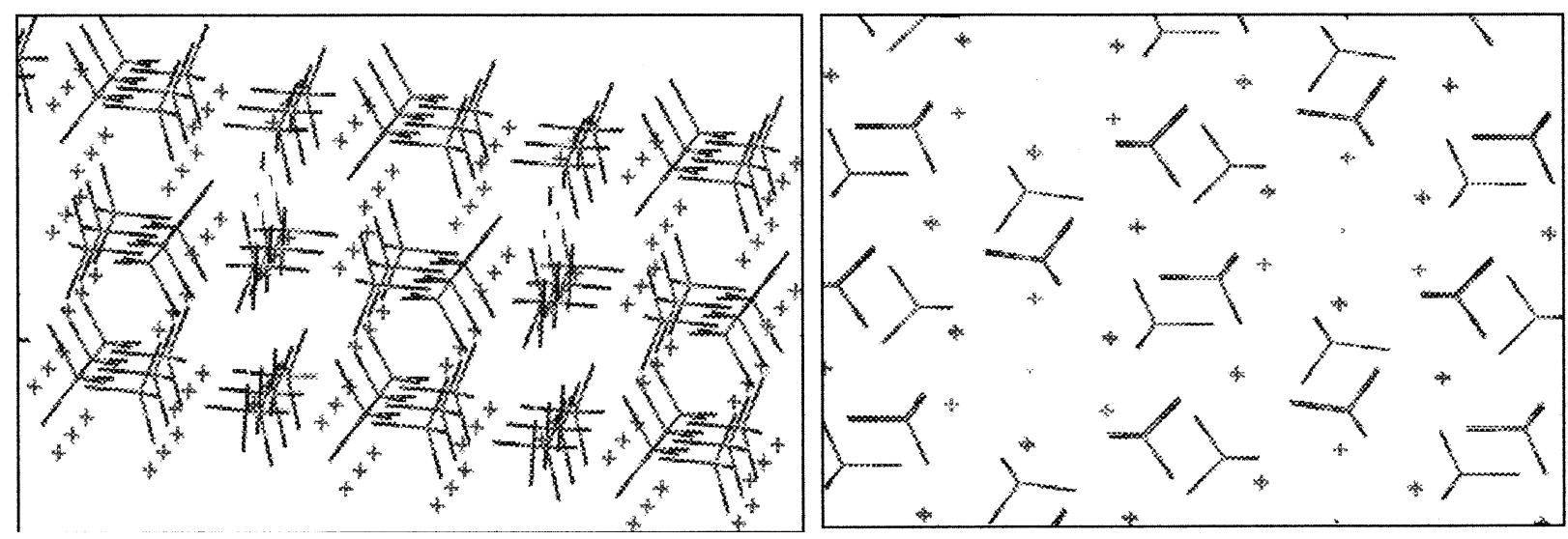

Fig. 3. Three-dimensional and top view of hydroxyapatite: + ca atoms; $>-$ phosphate groups. 


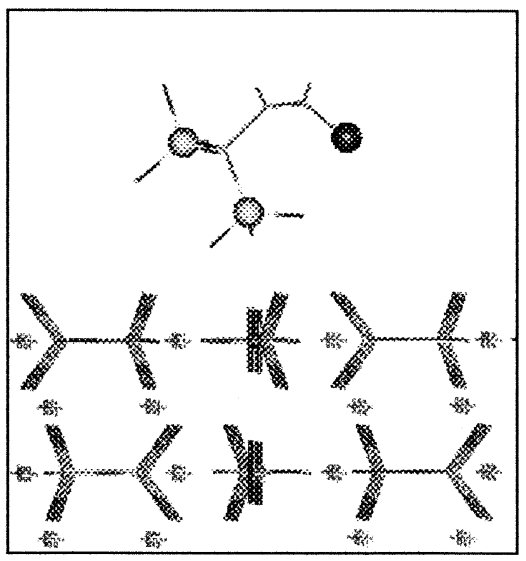

A
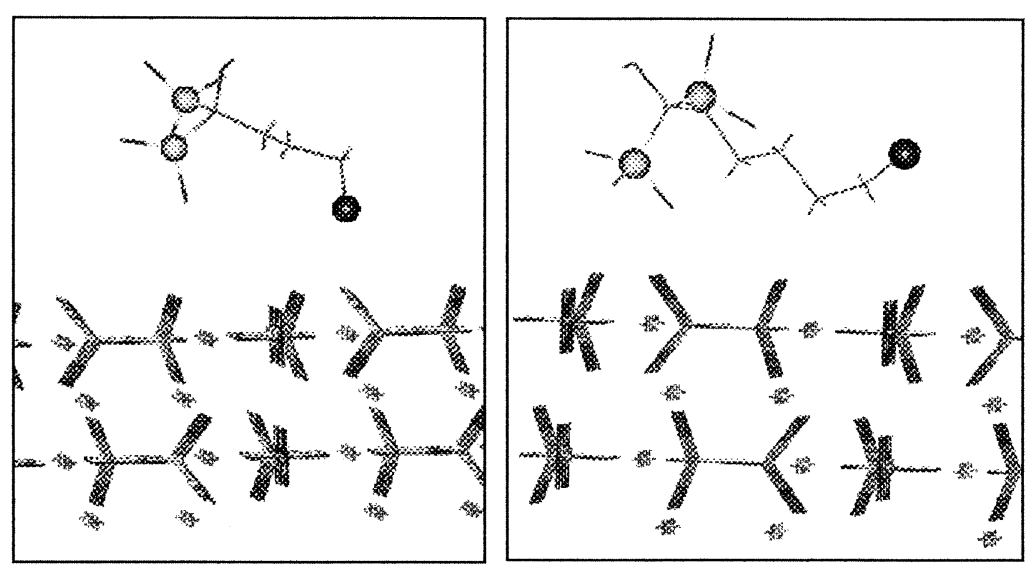

B

C

Fig. 4. Spatial view of the minimum energy complexes of pamidronate (A), alendronate (B) and neridronate (C) on the $(0,0,1)$ surface of hydroxyapatite.

(i) The most important intermolecular interactions occur between the phosphate and amino groups of BPs and the calcium ions of hydroxyapatite. Since both the oxygen atoms of the phosphate groups and the amino nitrogen atom (bearing one electron pair) shall have relatively large partial negative charges and the calcium atoms are positively charged, it can be expected that the dominant intermolecular interactions in the complexes are electrostatic in nature. The strength of these interactions is then strongly dependent upon the distance between the interacting charges, decreasing with the inverse of the square of the distance. Considering that the three BPs under study only differ in the length of their aliphatic chain bearing the amino group, it can be stated that, contrarily to the phosphate-calcium interactions (which shall not differ appreciably in the different complexes), the interaction between the amino group and the calcium ions of the matrix are unique for each bisphosphonate. The calculated distances between the nitrogen atoms and the nearest calcium atoms are $5.77 \AA$ for pamidronate, $3.75 \AA$ for alendronate, and $5.07 \AA$ for neridronate, implying stabilizing interaction energies that are similar for pamidronate and neridronate, but significantly more important for alendronate. The anti-resorption potencies relative to etidronate measured in vivo (in rats) were found to be $100 \times$, for both pamidronate and neridronate, and in between $100 \times$ and $1000 \times$ for alendronate [8]. Indeed, the pharmacologic activity of alendronate has been proved to be higher than those of pamidronate and neridronate, and alendronate has become established as a drug for treatment postmenopausal and other forms of osteoporosis [19,30]. Hence, the experimentally observed anti-resorption potencies exhibited by the three BPs studied correlate well with the calculated values for the corresponding amino-calcium interaction energy, indicating that this interaction may play a relevant role in determining the relative ability of BPs to act as anti-resorption agents.

(ii) The optimized structures of the BPs within the complex were found to be significantly different from those corresponding to the ground state conformer for the isolated BPs. In particular, in both the cases of pamidronate and alendronate, the intramolecular $\mathrm{OH} \ldots \mathrm{N}$ hydrogen bond which characterizes the most stable conformer of the isolated molecules does not remain in the surface-bound species, since the amino group is, as discussed, involved in the coordination with calcium. The energy difference between the conformation assumed by alendronate on the surface and that of the most stable conformer of the isolated molecule was calculated to be smaller than those obtained for the remaining two BPs. So, these results follow the same trend discussed in $i$ ) and is a further indication that alendronate has a structure that enables a better fit to the hydroxyapatite crystal preferred binding sites.

\subsection{Radiochemistry}

Radioactive preparations with radiochemical purity higher than $97 \%$, were identified as described. The ${ }^{153} \mathrm{Sm}-$ BPs complexes evaluated by the A system, migrate $(\mathrm{Rf}=$ $0.8-1.0$ ), while ionic ${ }^{153} \mathrm{Sm}$ and colloidal radioactive forms remains at the origin. The ${ }^{153} \mathrm{Sm}$-BPs complexes and the ionic ${ }^{153} \mathrm{Sm}$ evaluated by the $\mathrm{B}$ system, migrate $(\mathrm{Rf}=$ $0.8-1.0$ ), while colloidal radioactive forms remains at the origin. With these two systems it is possible to identify the three expected main radiochemical components: ${ }^{153} \mathrm{Sm}$-BPs complexes, ionic ${ }^{153} \mathrm{Sm}$ and radioactive colloidal forms 


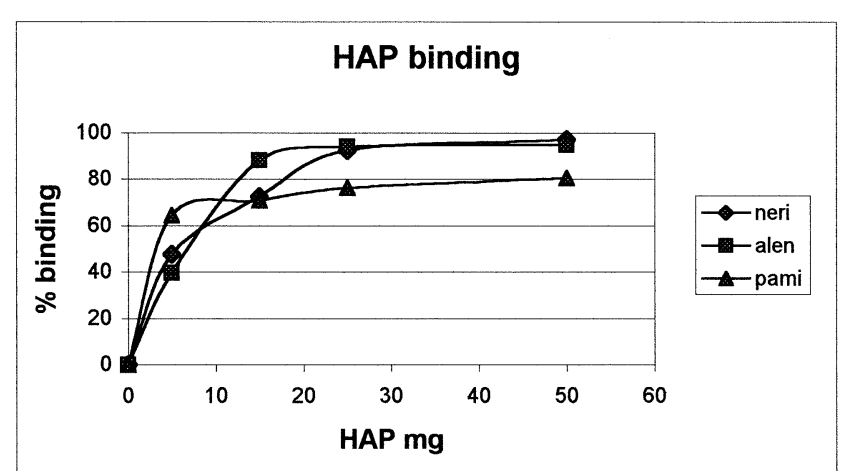

Fig. 5. Binding of ${ }^{153} \mathrm{Sm}$-pamidronate (pami), ${ }^{153} \mathrm{Sm}$-alendronate (alen) and ${ }^{153} \mathrm{Sm}$-neridronate (neri) to hydroxyapatite (HAP).

which could be considered as a mixture of hydroxy-des and/or neutral ${ }^{153} \mathrm{Sm}$ complexes.

\subsection{Hydroxyapatite binding}

The hydroxyapatite binding expressed as the percentage adsorption of ${ }^{153} \mathrm{Sm}$-pamidronate, ${ }^{153} \mathrm{Sm}$-alendronate and ${ }^{153} \mathrm{Sm}$-neridronate function of the amount of hydroxyapatite, is shown in Fig. 5. The adsorption curves have a similar profile as the described for ${ }^{99 \mathrm{~m}} \mathrm{Tc}$-bisphosphonates adsorption curves on tri-calciumphosphate [13]. The maximum percentage adsorption values, higher than $95 \%$, were achieved by ${ }^{153} \mathrm{Sm}$-alendronate and ${ }^{153} \mathrm{Sm}$-neridronate using amounts of hydroxyapatite higher then $25 \mathrm{mg}$, but the highest percentage adsorption for the minimum amount of hydroxyapatite was achieved by ${ }^{153} \mathrm{Sm}$-alendronate, $(\approx 90 \%$ versus $15 \mathrm{mg}$ ).

\subsection{Animal studies}

Skeletal uptake for ${ }^{153} \mathrm{Sm}$-pamidronate, ${ }^{153} \mathrm{Sm}$-alendronate and ${ }^{153} \mathrm{Sm}$-neridronate expressed in \% ID is shown in Fig. 6 . The highest value of skeletal uptake, was achieved at

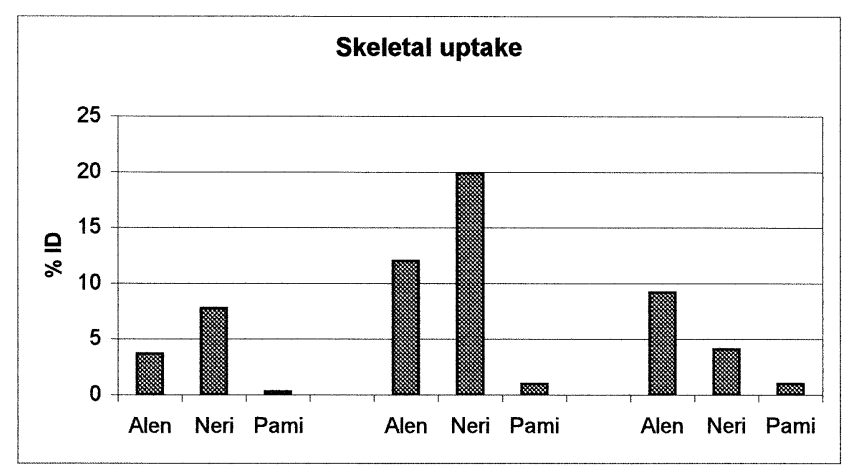

$1 \mathrm{H}$

$3 \mathrm{H}$

$24 \mathrm{H}$

Fig. 6. Skeletal uptake of ${ }^{153} \mathrm{Sm}$-pamidronate (Pami), ${ }^{153} \mathrm{Sm}$-alendronate (Alen) and ${ }^{153} \mathrm{Sm}$-neridronate (Neri) in mice, at 1,3 and 24 hours after injection.
3 hours after injection by ${ }^{153} \mathrm{Sm}$-neridronate $(19.9 \%)$, but at 24 hours the highest skeletal uptake value was observed for

${ }^{153} \mathrm{Sm}$-alendronate $(9.2 \%)$.

\section{Conclusion}

Molecular modeling studies on the isolated BPs, pamidronate, alendronate and neridronate, and on their hydroxyapatite complexes were undertaken. The molecular conformations adopted by the three BPs on the (001) surface of the hydroxyapatite crystal are markedly different from those corresponding to the isolated BPs, the most important structural changes being due to the need to satisfy the steric requirements for more favorable intermolecular interactions.

A good correlation could be established, between the experimentally available in vitro and in vivo experiments of the three ${ }^{153} \mathrm{Sm}$-BPs complexes and the corresponding interaction energies resulting from the Coulombic interaction, involving the nitrogen atom of the BPs and the nearest calcium atom of hydroxyapatite in the most stable BPhydroxyapatite complexes. In fact, the results of experimental hydroxyapatite binding and skeletal uptake of ${ }^{153} \mathrm{Sm}$-BPs complexes indicate that ${ }^{153} \mathrm{Sm}$-alendronate is preferred comparatively to ${ }^{153} \mathrm{Sm}$-pamidronate and ${ }^{153} \mathrm{Sm}$-neridronate. This is in agreement with the calculated BPs-calcium interaction energy that was shown by the molecular modeling studies to be significantly larger for alendronate than for the remaining BPs, as revealed by the shorter distance between the amino group and the nearest hydroxyapatite calcium atom observed in the alendronate-hydroxyapatite complex. These results seem to indicate that specific interactions involving the chain groups of the different BPs may play a relevant role in determining their relative ability to act as anti-resorption agents. On the other hand, they also point to the fact that the nature of the bisphosphonate-metal complexes do not play a decisive role in hydroxyapatite binding and skeletal uptake, confirming what was previous observed by others authors $[7,10,15]$. These conclusions are very important for the design of new BPs as drugs for bone diseases and as ligands to carrier radioactive metals. Also, from this work, it is also possible to conclude that molecular modeling studies are a good approach to evaluate specific interactions between BPs and hydroxyapatite and then it appears as a suitable method to be used in the design of new BPs with increased pharmacologic activity, and in the design of new bone therapeutic radiopharmaceuticals before in vitro and in vivo studies.

\section{Acknowledgments}

This work was supported by Fundação para a Ciência e Tecnologia (PRAXIS XXI, SAU/14036/98) and Schering Lusitana Lda. M. Neves and M. Chandia thank to ICCTI- 
Portugal and CONICYT-Chile the exchange scientists grants.

\section{References}

[1] B.L. Barnett, L.C. Strickland, Structure of disodium dihydrogen 1-hydroxyethylenediphosphonate tetrahydrated: a bone growth regulator, Acta Cryst. B35 (1979) 1212-1214.

[2] N.S. Black et al., Interactions at the organic/inorganic interface: binding motifs for phosphonates at the surface of barite crystals, J. Chem. Soc. Faraday Trans. 87 (1991) 3409-3414.

[3] S. Boissier et al., Bisphosphonates inhibit breast and prostate carcinoma cell invasion, an early event in the formation of bone metastases, Cancer Res. 60 (2000) 2949-2954.

[4] U. Burket, N.L. Allinger, Molecular Mechanics, ACS Monograph 177, Am. Chem. Soc., Washington, USA, 1982.

[5] Cerius-2 (Version 3.5), Molecular Simulations Inc., San Diego, CA, 1997.

[6] P.V. Coveney, W.J. Humphries, Molecular modelling of the mechanism of action of phosphonate retarders on hydrating cements, J. Chem. Soc. Faraday Trans. 92 (1996) 831-841.

[7] E. Deutsch, K. Libson, S. Jurisson, L.F. Lindoy, Technetium chemistry and technetium radiopharmaceuticals, Prog. Inorg. Chem. 30 (1983) 75-139.

[8] H. Fleisch, Bisphosphonates: mechanism of action, Endocrine Rev. 19 (1998) 80-100.

[9] H. Fleisch, R. Russel, M.D. Francis, Diphosphonates inhibit formation of calcium phosphate crystals in vitro and pathological calcification in vivo, Science 165 (1969) 1264-1266.

[10] M.D. Francis et al., Comparative evaluation of three diphosphonates: in vitro adsorption (C-14 labeled) and in vivo osteogenic uptake (Tc-99m complexed), J. Nucl. Med. 21 (1980) 1189-1189.

[11] O. Fromigue, L. Lagneau, J.J. Body, Bisphosphonates induce breast cancer cell death in vitro, J. Bone Min. Res. 15 (2000) 2211-2221.

[12] J.R. Green, Antitumor potential of bisphosphonates, Breast Cancer Res. 3 (Suppl. 1) (2000) A28.

[13] Y. Huigen et al., The absorption of ${ }^{99 \mathrm{~m}} \mathrm{Tc}(\mathrm{Sn})$ diphosphonate complexes on tri-calciumphosphate: the influence of preparation conditions, ligand type, incubation media and adsorption conditions. The reversibility of the adsorption, Appl. Radiat. Isot. 41 (1990) 189-194.

[14] M.J. Hwang, T.P. Stockfish, A.T. Hagler, Derivation of class 2. Derivation and characterization of a class II force field, CFF93, for the alkyl functional group and alkane molecules, J. Am. Chem. Soc. 116 (1994) 2515-2525.

[15] S.S. Jurisson, J.J. Benedict, R.C. Elder, R. Whittle, E. Deutsch, Calcium affinity of coordinated diphosphonate ligands. Single-crystal structure of $\left[(\mathrm{en})_{2} \mathrm{Co}\left(\mathrm{O}_{2} \mathrm{P}(\mathrm{OH}) \mathrm{CH}_{2} \mathrm{P}(\mathrm{OH}) \mathrm{O}_{2}\right)\right] \mathrm{ClO}_{4} \mathrm{H}_{2} \mathrm{O}$. Implications for the chemistry of technetium-99m-diphosphonate skeletal imaging agents, Inorg. Chem. 22 (1983) 1332-1338.

[16] M.I. Kay, R.A. Young, Crystal structure of hydroxyapatite, Nature 204 (1964) 1050-1052.
[17] A.R. Ketring, ${ }^{153} \mathrm{Sm}$-EDTMP and ${ }^{186}$ Re-HEDP as bone therapeutic radiopharmaceuticals, Nucl. Med. Biol. 14 (1987) 223-232.

[18] G.R. Kieczyowski et al., (4-amino-1-hydroxybutylidene) bisphosphonic acid sodium salt, MK-217 (alendronate sodium). An improved procedure for the preparation of 1-hydroxy-1,1-bisphosphonic acids, J. Org. Chem. 60 (1995) 8310-8312.

[19] U.A. Liberman et al., Effect of oral alendronate on bone mineral density and the incidence of fractures in postmenopausal osteoporosis, N. Engl. J. Med. 333 (1995) 1437-1443.

[20] K. Libson, E. Deutsch, B.L. Barnett, Structural characterization of a ${ }^{99} \mathrm{Tc}$-diphosphonate complex. Implications for the chemistry of ${ }^{99 \mathrm{~m}} \mathrm{Tc}$ skeletal imaging agents, J. Am. Chem. Soc. 102 (1980) 2476-2478.

[21] P. Masarachia, M. Weinreb, R. Balena, G.A. Rodan, Comparison of the distribution of $3 \mathrm{H}$-alendronate and $3 \mathrm{H}$-etidronate in rat and mouse bones, Bone 19 (1996) 281-290.

[22] A.J.B. McEwan, Use of radionuclides for the palliation of bone metastases, Semin. Radiat. Oncol. 10 (2000) 103-114.

[23] A. Mohamed, E. Deustch, W.R. Heinemam, Anion-exchange highperformance liquid chromatography of technetium-labelled phosphonoacetic acid skeletal imaging agents preparations, J. Chromat. 488 (1989) 463-469.

[24] G.R. Mundy, T. Yonada, T. Hiraga, Preclinical studies with zoledronic acid and other bisphosphonates on the bone microenvironment, Semin. Oncol. 28 (2001) 35-44.

[25] K.L. Nash, L.F. Rao, G.R. Chopin, Calorimetric and laser induced fluorescence investigation of the complexation geometry of selected europium gem-diphosphonate complexes in acidic solutions, Inorg. Chem. 34 (1995) 2753-2758.

[26] K.L. Nash, R.D. Rogers, J. Ferraro, J. Zhang, Lanthanide complexes with 1-hydroxyethane-1.1-diphosphonic acid: solvent organization and coordination geometry in crystalline and amorphous solids, Inorg. Chim. Acta 269 (1998) 211-223.

[27] S.R. Niketic, Kj. Rasmussen, The consistent force field: a documentation, lecture notes in chemistry, Vol. 3, Springer-Verlag, Heidelberg, Germany, 1985.

[28] M.J. Rogers et al., Molecular mechanism of action of bisphosphonates, Bone 24 (1999) 73S-79S.

[29] R.G.G. Russell, M.J. Rogers, Bisphosphonates: from the laboratory to the clinic and back again, Bone 25 (1999) 97-106.

[30] K.G. Saag et al., Alendronate for the prevention and treatment of glucocorticoid-induced osteoporosis, N. Engl. J. Med. 339 (1998) 292-299.

[31] W. Shakespeare et al., Structure-based design of an osteoclast-selective, nonpeptide Src homology 2 inhibitor with in vivo antiresorptive activity, Proc. Natl. Acad. Sci. 97 (2000) 9373-9378.

[32] D. Vega, R. Baggio, M.T. Garland, Monosodium 4-amino-1-hydroxy-1,1-butane-diyldiphosphonate trihydrate (alendronate), Acta Cryst. C52 (1996) 2198-2201.

[33] J.R. Zeevaart et al., Metal-ion specification in blood plasma incorporating the bisphosphonate, 1-hydroxy-4-aminopropilydenediphosphonate (APD), in therapeutic radiopharmaceuticals, J. Inorg. Biochem. 73 (1999) 265-272. 